\title{
A structural analysis of the situation in the Middle East in $1956^{1}$
}

\section{FRANK HARARY}

\section{Research Center for Group Dynamics, The University of Michigan}

\section{Résumé}

We attempt to display a systematic approach for the distinction between states of equilibrium and disequilibrium in the interrelationships between nations, using as corroborative material the rapid shifts in $\mathbf{1 9 5 6}$ among the various nations, brought about by the Middle Eastern situation. The psychological theory behind this approach is that of structural balance, which is pertinent in the present context to balance of power, while the logical framework involves the mathematical theory of graphs. We do not assert that this theory in its present form is predictive, but we do feel that it offers a wellorganized mode of thinking which, although simple, may be fruitful. We also comment on some aspects of the Hungarian situation in terms of structural balance. It must be borne in mind throughout this article that it was written in early 1957 and that therefore the interrelationships among nations described herein reflect that time period.

\section{Introduction}

The theory of structural balance (1) has been tested in the psychological laboratory

\footnotetext{
${ }^{1}$ I am indebted to Anatol Rapoport for suggesting this article and to Dorwin Cartwright for making several helpful comments.
}

(10) for groups of persons. It was anticipated by Heider (8) and Newcomb (II) with their "tendency toward balance" and "strain toward symmetry," respectively. More recently, an axiom system based on balance theory has been proposed (2) from which the assertions of Freud (3) regarding the development of the ego of an individual can be logically derived. From the general approach of "systems theory" (9), the notion of balance already experimentally verified (10) for groups of individuals, and then applied (2) to the structure of the ego of a single individual, might well be considered for larger systems than groups. This is similar in spirit to the work of Guetzkow (4). The purpose of this note is to propose the mathematical theory of balance as a systematic approach to the interaction between nations.

We set the stage for the later discussion of the international situation in terms of the theory of structural balance by presenting a brief outline of this theory on an intuitive level. Fortunately, it is not necessary to become steeped in the mathematical details of the theory of graphs, but it is sufficient to be able to follow the argument with the assistance of the accompanying figures. In succeeding sections we take up a rationale for applying balance theory to nations, examples of the tendency toward balance, the global 
situation, desires and perceptions, the Hungarian developments, and a prediction attempt.

In the applications of balance theory to relationships between pairs of nations, our discussion does not contain any conclusions which are not deducible from a simple common sense postulate of polarity. Such a polarity principle would assert that all nations must be divided into two coalitions in such a way that any two nations in the same coalition are friendly and any two nations from different coalitions are unfriendly. This is very closely related to, but not completely identical with the structural theorem for balance which appears in the next section. The principal difference lies in the word "structure." For there may be indifference relationships between certain pairs of nations which lie either in the same coalition or in different coalitions. The polarity approach does not make this distinction. At present, balance theory has not as yet been developed to the extent that it enables the handling of structural situations in which the international bonds may have varying strength. It is precisely this open problem area which holds the greatest promise for the evolvement of a theory which would be sufficiently comprehensive to be predictive. It is very likely that the mathematics of probability theory will be involved.

\section{Balance Theory}

We now describe briefly the historical background of balance theory and then discuss the modification for generalizing this theory to nations from those entities for which it has already been shown to be appropriate as a mathematical model. We begin by quoting a paragraph from Cartwright and Harary (1), which sets the stage for the generalization of Heider's theory from a triad consisting of two persons and one object to any number of entities regardless of the number of persons and the number of objects.
Heider (8), reflecting the general field-theoretical approach, has considered certain aspects of cognitive fields which contain perceived people and impersonal objects or events. His analysis focuses upon what he calls the $P-O-X$ unit of a cognitive field, consisting of $P$ (one person), $O$ (another person), and $X$ (an impersonal entity). Each relation among the parts of the unit is conceived as interdependent with each other relation. Thus, for example, if $P$ has a relation of affection for $O$ and if $O$ is seen as responsible for $X$, then there will be a tendency for $P$ to like or approve of $X$. If the nature of $X$ is such that it would "normally" be evaluated as bad, the whole $P-O-X$ unit is placed in a state of imbalance, and pressures will arise to change it toward a state of balance. These pressures may work to change the relation of affection between $P$ and $O$, the relation of responsibility between $O$ and $X$, or the relation of evaluation between $P$ and $X$.

We now quote from Heider (8), whose basic hypothesis asserts that there is a tendency for cognitive units to achieve a balanced state. Pressures toward balance may produce various effects:

In the case of two entities, a balanced state exists if the relation between them is positive (or negative) in all respects. ... In the case of three entities, a balanced state exists if all three relations are positive in all respects, or if two are negative and one positive.

If no balanced state exists, then forces towards this state will arise. Either the dynamic characters will change, or the relations will be changed through action or through cognitive reorganization. If a change is not possible, the state of imbalance will produce tension.

In the article, "Structural Balance: A Generalization of Heider's Theory" (1), there is a brief summary of some of the major results of the work of others who used Heider's theory as a point of departure for further theoretical and empirical work.

In Cartwright and Harary (2), there is provided an application of balance theory in which the entities are no longer persons and objects as in Heider's theory, but are parts of a person's personality, such as ego and id, as well as "inner instinctual stimuli" together 
with external stimuli, both pleasant and unpleasant. It is shown in (2) that the assertions of Freud (3) are derivable as logical consequences of an axiom system which includes as one of its postulates the "tendency toward balance" which is stated as an empirical hypothesis in the next section.

We will assume throughout this paper that nations are admissible entities for study by the approach of the theory of structural balance and that the relation of alliance, cooperation, agreement, or other friendly behavior between a pair of nations is intrinsically positive. This approach was implicitly anticipated by Guetzkow (4). On the surface, the conclusions to be drawn from this way of presenting international relations are not surprising. Any common sense analysis would yield the same results. Of course it is desirable that a first rough approximation to reality using a mathematical model be consistent with common sense results. The principal idea which makes this approach more than a collection of diagrams to portray a known situation is that the structure of international relations among several nations or groups of nations is made explicit. The particular manner in which nations are related to each other may be positive, negative, indifferent, or even sometimes ambivalent. This is discussed in more detail in a later section which explicitly spells out several of the limitations of this theory in its present stage of development.

\section{Tendency Toward Balance}

A graph (7) can be represented geometrically by a set of points in the plane together

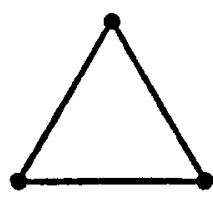

a

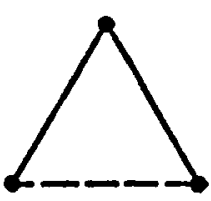

b

FIGURE 1

with some of the lines joining pairs of points. Thus the graph of Figure 1a has three points and three lines. A signed graph (Figure 1) is a generalization of a graph in which some of the lines are regarded as positive and the remaining lines as negative. Signed graphs are useful in providing a structural depiction of a binary relation with both positive and negative relationships (lines) between the pairs of objects or entities (points) involved. The realization of signed graphs in the present article takes points as nations, positive lines as friendship or alliance, and negative lines as hostility or warfare. For example, the four signed graphs of Figure 1 (in which solid lines are positive and dashed lines are negative) contain $0,1,2$, and 3 negative lines respectively.

A signed graph is balanced if, and only if, all of its "cycles" are positive. The importance of this concept lies in the following:

\section{EMPIRICAL HYPOTHESIS (Tendency}

\section{Toward Balance)}

A balanced structure has greater stability than one which is not balanced. If a given structure is not balanced, there will be a tendency to modify the structural bonds in order to achieve balance. There is both empirical and theoretical justification (1) for this hypothesis. Other related tendencies are discussed in the article (6). These include tendencies toward clustering, completeness, and positivity:

Tendency Toward Clustering. If a collection of entities, having a relationship which is intrinsically positive, has achieved balance, and if, furthermore, one of these entities is able to relo-

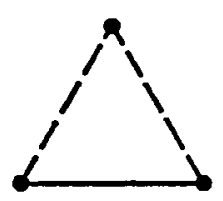

C

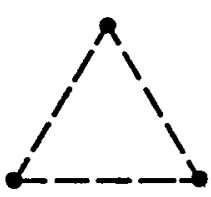

d 
cate the remaining ones into subgroups, then the entities will tend to be realigned into two clusters in accordance with the structure theorem for balance.

Tendency Toward Completeness. A group structure will tend toward completeness, that is, if two entities are not yet interrelated in the structure, then a bond will tend to be induced to appear between them.

Tendency Toward Positivity. If a relationship is intrinsically positive, then any individual in a group will tend to show a marked preference to forming positive bonds rather than negative ones.

We note the emphasis in the statement of these last three tendencies that the relationship under consideration must be intrinsically positive. We have assumed that alliance or other cooperation or agreement between a pair of nations is intrinsically positive.

In order to make the definition of structural balance precise, we require the following concepts. A cycle of a graph is a collection of lines of the form $A_{1} A_{2}, A_{2} A_{3}, \ldots, A_{n-1} A_{n}$, and $A_{n} A_{1}$; the length of a cycle is the number of lines in it. Thus each of the signed graphs of Figure 1 consists of one cycle of length 3 . The sign of a cycle is the product of the signs of its lines. Thus a cycle is positive if and only if it has an even number of negative lines; the cycles of Figure $1 \mathrm{a}$ and $1 \mathrm{c}$ are positive while those of Figures $1 b$ and $1 d$ are negative. The basic theorem concerning balance is the:

\section{STRUCTURAL THEOREM FOR BALANCE}

A signed graph is balanced if and only if its points can be separated into two mutually

to

$t_{1}$

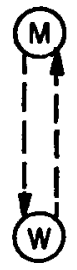

exclusive sets $S_{1}$ and $S_{2}$ in such a way that each positive line joins points of the same set while each negative line joins two points from different sets.

This theorem gives insight into the nature of structural balance and in addition provides a characterization of balance which is of assistance in recognizing balanced structures.

In a directed graph, the lines are directed and $\overrightarrow{A B}$ denotes the line from $A$ to $B$. The convention (1) with regard to balance of a directed signed graph is that the direction of the lines be ignored.

In the next two sections, we represent changing situations by a sequence of signed graphs which present the structure at different times: $t_{0}$ (initial time), $t_{1}, t_{2}$, etc. These times are chosen in such a way that some structural transformation has occurred between $t_{0}$ and $t_{1}$, between $t_{1}$ and $t_{2}$, etc. We illustrate this kind of graphical representation with an anecdote:

$A$ man $M$ and his wife $W$ were quarreling (at time $t_{0}$ in Figure 2) when a bystander B intervened and tried to make peace (at $t_{1}$ ) whereupon man and wife stopped arguing and both turned on the bystander (at $t_{2}$ ).

We note that at $t_{0}$ there is balance since the cycle of length 2 has both lines negative and thus this cycle is positive. Then at $t_{1}$ we find a negative cycle of length 3 so that the structure is unbalanced and (in accordance with our empirical hypothesis) less stable. Finally at $t_{2}$, balance has been attained by changing

$t_{2}$
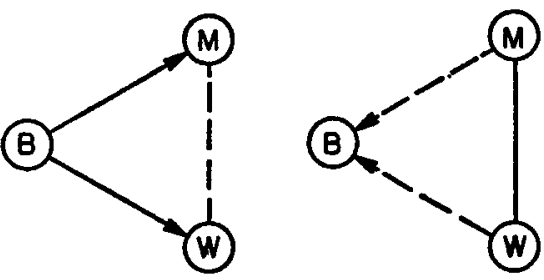

Figure 2 
to

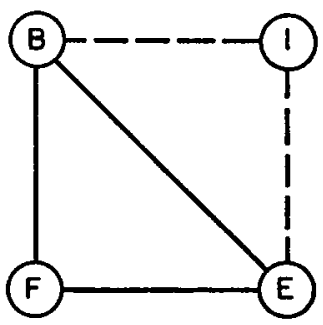

$t_{1}$

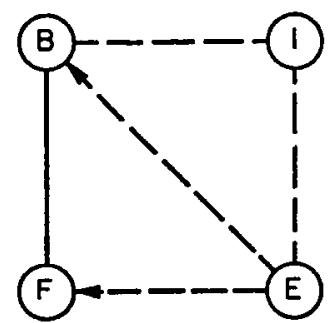

Figure 3 $t_{2}$

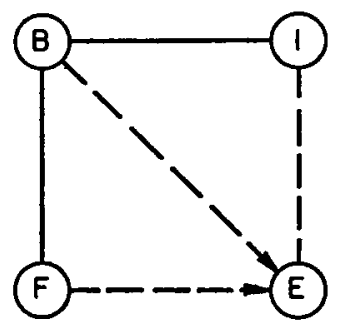

the structure. We shall now see several similar situation sequences among nations.

\section{Tendency Toward Balance} in the Middle East

We use the following abbreviations in the diagrams presenting the dynamics of international relationships; and also in the accompanying discussion.

$\begin{aligned} \text { E } & \text { Egypt } \\ \text { A } & \text { the other Arab countries } \\ \text { B } & \text { Great Britain } \\ \text { C } & \text { Canada } \\ \text { F } & \text { France } \\ \text { I } & \text { Israel } \\ \text { D } & \text { India } \\ \text { U } & \text { U.S.A. } \\ \text { R } & \text { U.S.S.R. } \\ \text { H } & \text { Hungary }\end{aligned}$

The following developments are pictured in Figure 3. Some time ago (at $t_{0}$ ), B and $F$ were both acting in a friendly way toward $\mathrm{E}$, which was quite hostile to I, which in turn was inclined to feel a disliking for $\mathrm{B}$ for this and other reasons. Then $E$ confiscated some important property belonging jointly to $B$ and $F$ (at $t_{1}$ ). The result was that $B$ and $F$ attacked $\mathrm{E}$, and I also attacked $\mathrm{E}$.

President Eisenhower then expressed explicit disapproval of these attacks. The next three figures are accompanied by the following three excerpts (respectively) from the front page of the Ann Arbor News of November 3, 1956.

AMMAN, Jordan-(U.P.) - King Hussein of Jordan sent a warm message of thanks to President Eisenhower for his support of "right and justice" in the Middle East conflict.

CALCUTTA, India-(U.P.)-The United States' stand in the Middle East dispute today stirred an unprecedented wave of pro-Americanism among the Indian press and public.

"Indian, American-bhai bhai [brothers]," was a commonly heard slogan.

USSR Woos Syria, Blasts Britain, France

MOSCOW-(AP) - The Soviet Union late today offered Syria "every assistance" and turned to

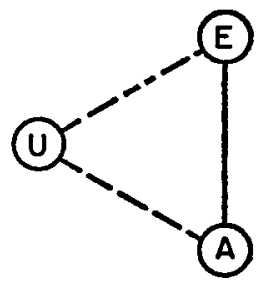

$t_{1}$

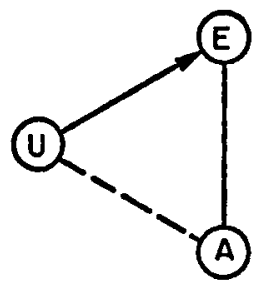

Frgure 4

\section{$t_{2}$}

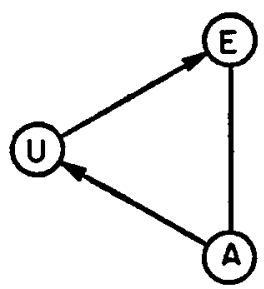


$t_{0}$

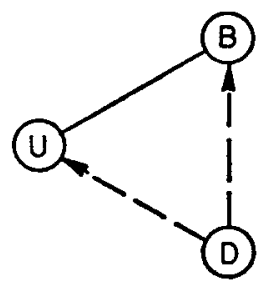

$t_{1}$

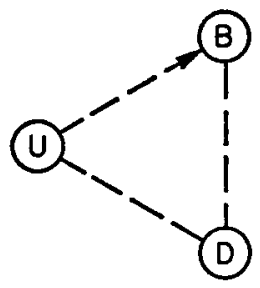

Figure 5
$T_{2}$

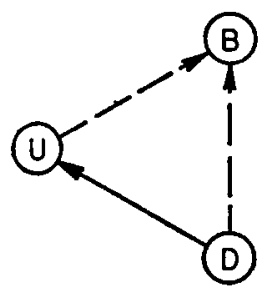

heavy propaganda weapons on Britain, France and Israel.

It appeared here the Soviet Campaign had two main targets:

1) To destroy any remaining friendship Britain and France have in the Middle East and Asia.

2) To divert the world's attention from developments in Hungary.

The Hungarian situation, mentioned here en passant, will be the subject of a later section.

In one sense, the three scenes of Figure 6 are in incorrect chronological order and are better represented as in Figure 7. Here B and $R$ are inimical at $t_{0}, R$ builds up the position of $E$ at $t_{1}$, and active hostility between $B$ and $E$ is induced at $t_{2}$.

\section{The Global Situation}

We now present the combined structure of which the previous figures are fragments or substructures. Since the time instants $t_{0}, t_{1}$, and $t_{2}$ are different for these various figures, the combined structure is presented in more than three scenes. By Figure 8.0, 8.1, . . we mean Figure 8 at time $t_{0}, t_{1}, \ldots$ respectively. We restrict these figures to the eight points $A, B, D, E, F, J, R$, and $U$ for relative simplicity. In Figure 8.0 we have the fairly static situation as it was before the recent events.

This is a balanced configuration in which, in accordance with the Structural Theorem, the two disjoint sets are given by $S_{1}=\{D, I, R\}$ and $S_{2}=\{A, B, E, F, U\}$. At this stage, both $B$ and $U$ were building up the position of $E$ with armaments and both direct and indirect economic assistance, which induced negative feelings from I to B and U. This situation, although balanced, was a strange one since it found $I$ in the same camp or coalition with $D$ and $R$ not because of any explicit or implicit friendship, but because they shared the same dislikes.
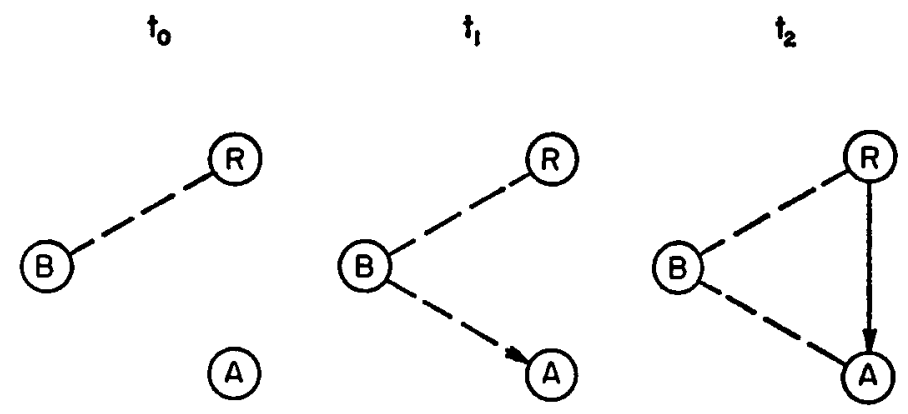

Figure 6 
to

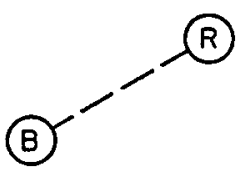

(E) $t_{1}$

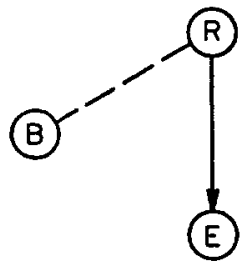

$t_{2}$

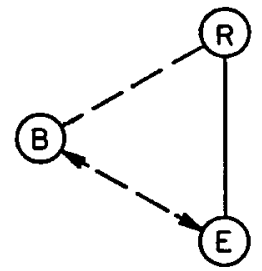

Figure 7

In Figure 8.1, the only change is the addition of the positive lines $\overrightarrow{R E}$ and $\overrightarrow{R A}$ as realized in the form of bartering Czech and Russian armaments for Egyptian cotton (with military advice included in the bargain). This has the effect of creating a situation which is not balanced since (among others) the cycles ERU and BER are negative.

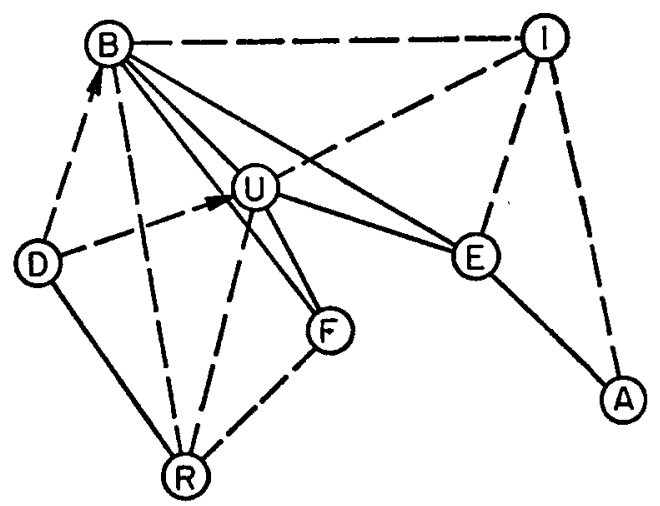

Figure 8.0

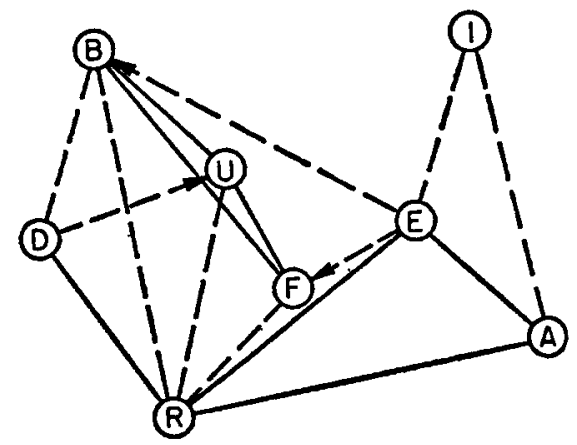

Figure 8.2
Egypt's nationalization of the Suez Canal is depicted in Figure 8.2, while the subsequent military attack on $E$ by $I$ and shortly thereafter by $B$ and F, together with the induced positive lines (in accordance with the Empirical Hypothesis) are shown in Figure 8.3.

Both of these are balanced structures;

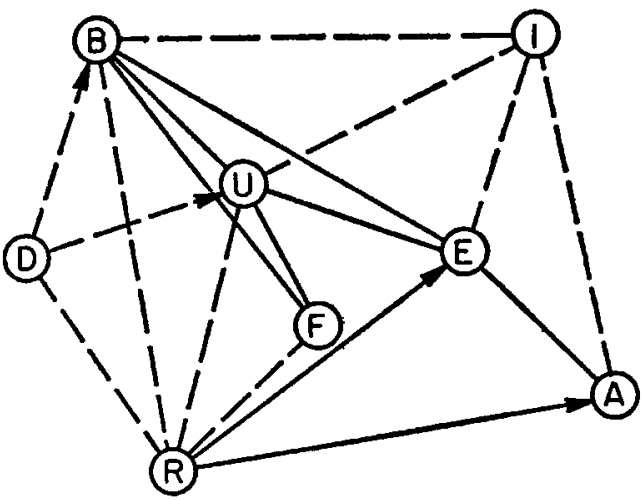

Figure 8.1

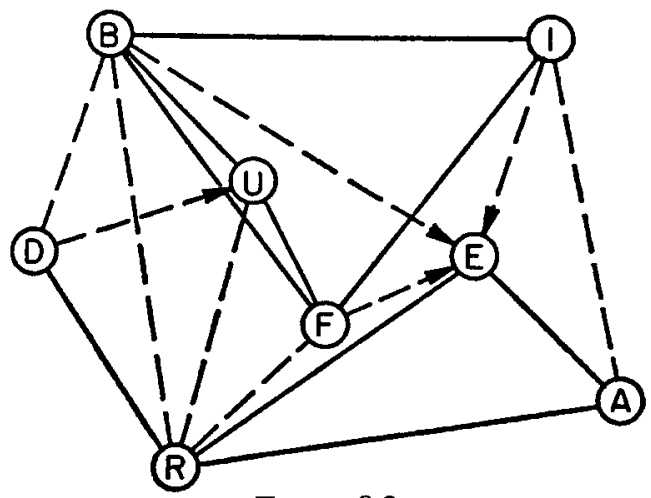

FIGURE 8.3 


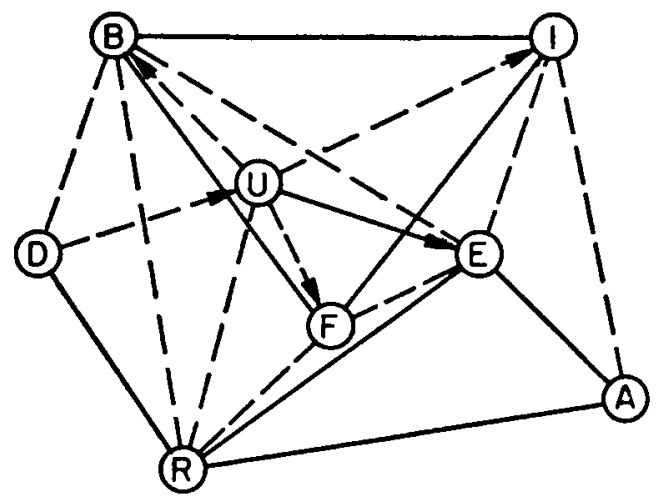

Figure 8.4

furthermore they have the same coalitions: $S_{1}=\{B, F, I, U\}$ and $S_{2}=\{A, D, E, R\}$. The main structural difference (not displayed diagrammatically) is that the bonds have become more intense in the latter figure. It is interesting to note that this situation, although balanced, is not a pleasant one for $U$ since the other coalition finds both $R$ (with its strong negative valence for $U$ ) and $A, E$ (the controllers of an important commodity in U's economy) together. Because of this fact, as well as the ethical and moral issues involved in an unannounced attack on one weaker nation by two stronger ones, we have in Figure 8.4 the American denunciation of the attack on $E$ by $B$ and $F$ as well as by $I$.

This situation is not balanced since the cycles FRU and BDU are negative. In fact all the negative cycles of Figure 8.4 contain U! The only change from this to Figure 8.5 is the vigorous reversal of sign from negative to positive of the directed line from $D$ to $U$.

Thus the cycle BDU is now positive. However, as a result of the definite change in sign of the line DU, the cycle DRU is now negative. A natural prediction is that the considerable tension felt by the Indian government as a result of this negative cycle DRU will cause $D$ to consider a change in sign in the line $\overrightarrow{D R}$ from positive to negative.

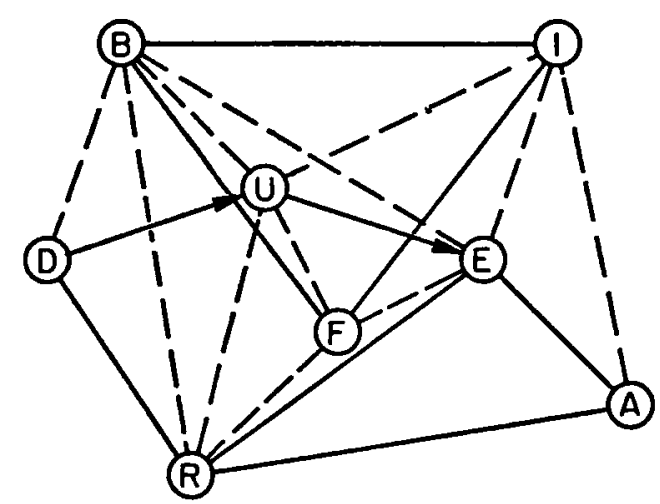

Frgure 8.5

\section{Desires and Perceptions}

It seems clear that the balanced graph of Figure 8.3 represents fairly accurately the desires of $R$ for a stable coalition. On the other hand the wishes of $U$ would not be too badly reflected in a balanced graph which (from among the eight nations of Figures 8.0 through 8.5) has coalitions: $S_{1}=\{R\}$ and $S_{2}=\{A, B, D, E, F, I, U\}$. The somewhat unrealistic aspect of this possibility is that it has $I$ in the same coalition with $A$ and $E$.

However, this is precisely reflected in the following quotation by former President Truman as reported in Drew Pearson's column of January 5, 1957.

I would make Israel the industrial country of the Near East, then let the Arabs raise crops to feed Israel and themselves. They're cousins. They don't have to fight. They're all semitic peoples.

It is clear that this approach involves the difficult task of persuading both I and $A$ to replace their strong negative bond by a decisively positive one. From page 13 of the Hadassah Newsletter of November, 1956, which certainly gives the Israeli viewpoint, we can infer even from the headline alone that the essential difficulty lies in the transformation of the sign of the directed line $\overrightarrow{\mathrm{AI}}$ 


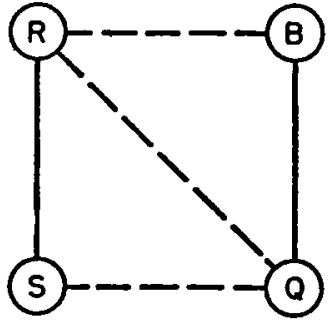

Figure 9

from negative to positive, since I appears to be ready for a positive $\overrightarrow{\mathrm{IA}}$ line just as soon as it can be arranged.

\section{Israel Seeks Permanent Peace}

The immediate results of Israel's action were the following: The imbalance of power in the Middle East, caused by the Soviet arming of Egypt, has been corrected, and the consequent fear of an attack on Israel has been minimized. Nasser's ambitions for hegemony over the Arab world and the elimination of Israel received a set-back, perhaps permanently. Not only Israelis but many of the leaders of Arab states may now breathe easier.

The last sentence of this quotation has other interesting implications pertaining to the fact that some of the weaker Arab nations also felt threatened by the possibility of Egyptian domination.

Let us denote $S=$ Syria, $Q=$ Iraq, and $J=$ Jordan. Then actually $A=$ the Arab nations other than Egypt $=\{S, Q, J, \ldots$.$\} . With$ increased Soviet influence in Syria, there is a balanced situation between $Q, R$, and $S$ as shown within Figure 9 and as observable by certain Syrian threats directed toward Iraq. Partly because of her willingness to continue to accept financial support from Great Britain, Iraq is generally regarded as the most westerly oriented of the Arab nations. This is also displayed in the balance of Figure 9.

The perception of the situation by Egypt is nicely summarized in the following quotation from the front page of a December, 1956, issue of the Ann Arbor News:

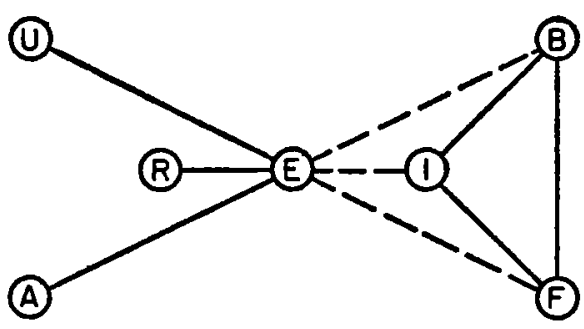

Figune 10

CAIRO, Egypt-(AP) - President Nasser declared today the United States and Soviet Russia "were with us" when Egypt defended herself against British, French, and Israeli attacks. He denied his Arab allies had failed him.

This is shown in the balanced structure of Figure 10, in which the coalitions are: $S_{1}=\{B, F, I\}$ and $S_{2}=\{A, E, R, U\}$.

Another, perhaps even more striking demonstration, of differences in perception took place before B, F, and I agreed to withdraw their troops. $F$ or $R$ proposed to $U$ at that time that these two most powerful nations join in evicting $B, F$, and $I$ by the use of their superior military might. This proposal was summarily rejected by $U$ as "unthinkable." Figures 11 and 12 represent this situation as perceived by $R$ and $U$ respectively. In each of these figures, the proposal of $R$ is made at time $t_{1}$ and the reaction of $U$ is given at $t_{2}$, and in each of them a structural situation which was upset by the proposal of $\mathbf{R}$ was restored.

\section{The Hungarian Situation}

We find in the Hungarian situation examples of two different kinds of structural changes. These are also amenable to analysis in the framework of structural balance. The first is the strengthening of an already existing bond without changing its sign. This is shown in Figure 13, in which two lines of the same sign between the same pair of nations indicate a stronger bond than one line. Here the developments involving the free world 
to

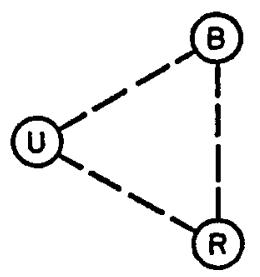

$t_{0}$

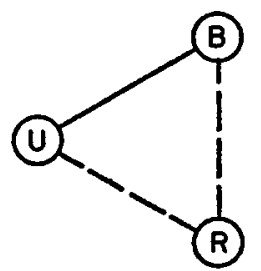

$\dagger_{1}$

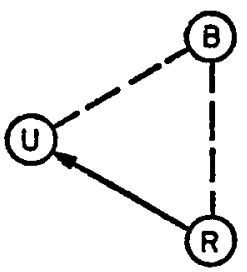

Figure 11

$t_{1}$

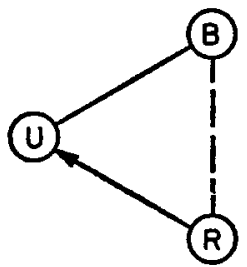

Figure 12 $t_{2}$

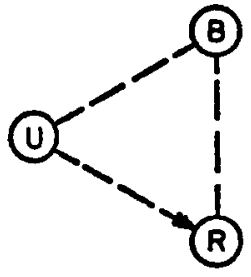

$t_{2}$

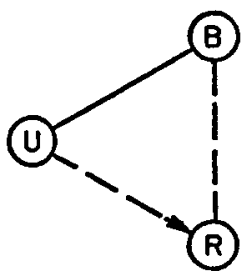

$\mathrm{FW}=\mathrm{B}, \mathrm{F}, \mathrm{U}, \mathrm{C}$, etc.; $\mathrm{H}=$ Hungary; and $\mathrm{R}=$ the U.S.S.R. are depicted. Each of the signed graphs of Figure 13 is balanced, but intuitively at $t_{2}$, the situation is "most balanced."

The second kind involves the splitting of a point, in this case the point $D=$ India, into two points, namely DG for the Indian government and DP for the Indian people. At $t_{0}$ we find (in Figure 14) India and the U.S.S.R. on friendly relations while the people of Hungary are making their try for freedom. This attempt is forcefully suppressed at $t_{1}$. Then at $t_{2}$ the official Indian government reacts to this suppression by condoning the act, but the people of India feel very differently and criticize the U.S.S.R. for its actions and their Indian government for its stand in letters to the editors of Indian newspapers and in other well-publicized ways. The inevitable result of this pressure of Indian public opinion is realized at $t_{3}$, when the Indian government reluctantly makes an official anti-Soviet statement. When the two points DG and DP are then coalesced into the single point $D$, we obtain the balance graph at time $t_{4}$. We note $t_{0}$

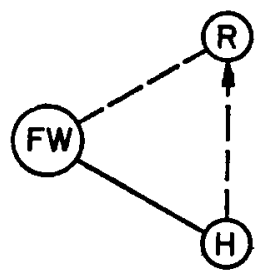

$t_{1}$
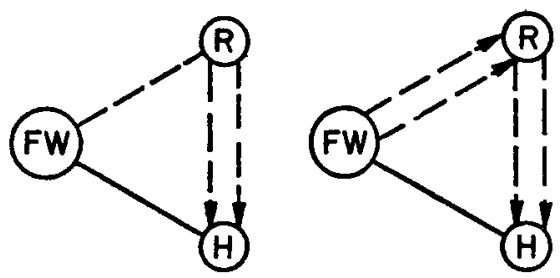

Figune 13 


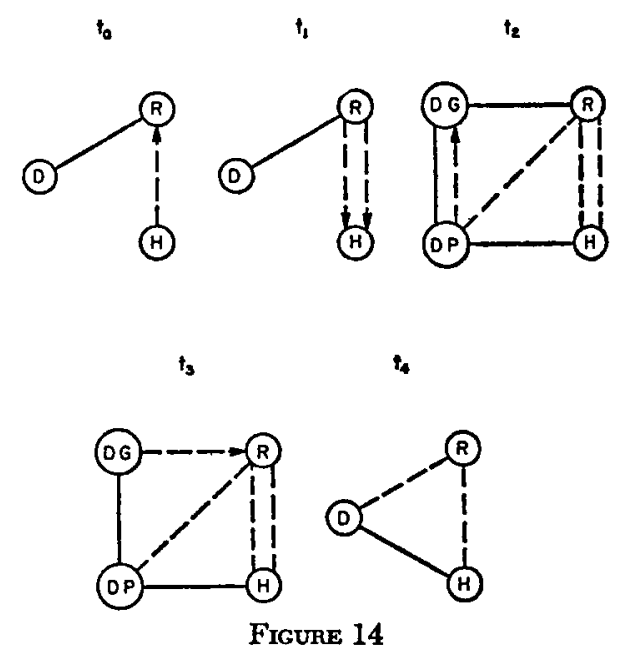

that at time $t_{2}$, the phenomenon of ambivalence $(3,5)$ was in effect between DP and DG, with both a positive line and a negative line joining these two points. Here the positive line stands for the "unit" relation of ownership or identification while the negative line gives the "sociometric" relation of opinion on the like-dislike scale.

\section{Limitations of the Theory}

We list here some of the conceptual problems whose solutions would strengthen the theory of structural balance both generally and with regard to the kind of applications and representations discussed in this article. These show that we have indulged in considerable over-simplification as a first approximation.

1. We do not yet have a really useful model or accurate measurement of the degree of intensity of a bond between two points. One could denote by the numbers $1,1 / 2,0$, $-1 / 2$, and -1 the relations loving, liking, indifference, disliking, and hating respectively. But this merely provides a possible beginning for the problem.

2. In the graphical representation of the various events discussed here, there was no distinction made between the lines standing for overt and covert acts, or for power as opposed to international sociometric choice or friendship. This problem calls for far more than a pictorial distinction which, of course, can be given by using lines of various colors. Namely, it is the operational interdependence of the various relationships which is important.

3. No distinction was made between durable underlying bonds and transient ones. For example, most observers would agree that regardless of the present status of the bond between $B$ and $U$, it will ultimately revert to its previous traditional strong positivity.

4. The points used to represent nations and groups of nations are themselves signed graphs. We have already seen two examples of this phenomenon in microscopic view of the point $D$ in Figure 14, and that of $A$ in Figure 9. This does not necessarily mean that these two points decomposed or fell apart, but only that they are examined more closely. In terms of systems theory, we may ask how does the structure of its subsystems affect the behavior of a system in its interaction with other systems?

\section{The Prediction Problem}

Even with the available theory, however, one can still attempt to predict from the present situation, which is fairly represented in Figure 8.5. No one will argue that regardless of what two coalitions (if any) emerge, $U$ will be in one and $R$ will be in the other. There is also no doubt that $B$ and $F$ will be in the same coalition with $\mathrm{U}$. There appears to be more and more evidence that eventually, $\mathrm{D}$ will choose to be in the same camp with U. The visit of King Saud to the U.S.A. is a clear demonstration of the intent of $U$ to break up the point $A$ and carry as many of its components as possible into the coalition with $\mathrm{U}$.

With their present essentially dictatorial 
controls, both $\mathrm{E}$ and $\mathrm{S}=$ Syria appear to be firmly in the coalition containing $R$. The only one of the eight points in the six scenes of Figure 8 not yet mentioned is I=Israel. This is a critical problem, and it takes more than a mathematical theory to achieve a permanent peace. We shall not attempt to predict on this point, other than to note that from all appearances, $R$ has no desire to have $I$ in its coalition.

The systematic structural balance approach of this article offers a clear approach to the study of international situations. One can draw the signed graph of a given state of events and examine it for balance. If it is balanced there will be a tendency toward the status quo. If it is not balanced, one should examine each of the bonds between the pairs of nations in a cycle with regard to relative strength in the situation. One might then predict that the weakest such bond will change sign.

\section{REFERENCES}

1. Cartwright, D., and Harary, F. "Structural Balance: a Generalization of Heider's Theory," Psychological Review, 63 (1956), 277-93.

2. —. A Note on Freud's "Instincts and
Their Vicissitudes," International Journal of Psychoanalysis, 40 (1960), 287-90.

3. Fneud, S. "Instincts and Their Vicissitudes," First published in Internationals Zeitschrift für artztliche Psychoanalyse, Vol. 3, 1915. Translation by C. M. BAINES in Collected Papers, Vol. 4, Metapsychology, London: The Hogarth Press, 1925.

4. GuetzKow, H. "Isolation and Collaboration: a Partial Theory of Internation Relations," The Journal of Conflict Resolution, 1 (1957), 48-68.

5. Harary, F. "Structural Duality," Behavioral Science, 2 (1957), 255-65.

6. "On the Measurement of Structural Balance," Behavioral Science, 4 (1959), 316-23.

7. Harary, F., and Norman, R. Z. Graph Theory as a Mathematical Model in Social Science. Ann Arbor, Mich.: Institute for Social Research, 1953.

8. Hemer, F. "Attitudes and Cognitive Organization," Journal of Psychology, 21 (1946), 107-12.

9. Mirlek, J. G. "Toward a General Theory for the Behavioral Sciences," American Psychologist, 10 (1955), 513-31.

10. Morissetre, J. O. "An Experimental Study of the Theory of Structural Balance," Human Relations, 11 (1958), 239-54.

11. Newcoms, T. M. "An Approach to the Study of Communicative Acts," Psychological Review, 60 (1953), 393-404. 